\title{
Review
}

\section{Advances in Nanotechnology: Influence on Biomolecular Detection Sensors}

\author{
Khalid Mahmood Arif ${ }^{a b *}$, Kutay Icoz ${ }^{b}$ and Ijaz Ahmad Chaudhry ${ }^{a}$ \\ ${ }^{\mathrm{a}}$ Mechatronics and Control Engineering, University of Engineering and Technology Lahore-54890, Pakistan

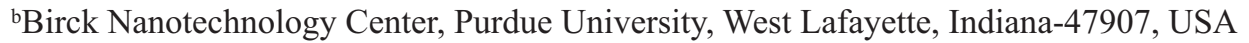 \\ (received April 6, 2012; revised February 8, 2013; accepted February 26, 2013)
}

\begin{abstract}
Nanodevices and biomolecules have incredibly strong correspondence in terms of size and physical properties. In this review, three major types of nanodevices, namely cantilevers, nanowires and carbon nanotubes, have been discussed and how they have resulted in new sensor designs or helped push the limits of detection in existing schemes. After brief overview of each type and the ways it could be used in biosensing, recent research efforts are presented to emphasise the challenges and achievements in that particular category.
\end{abstract}

Keywords: nanobiosensors, biosensors, nanodevices, cantilevers, nanowires, carbon nanotubes

\section{Introduction}

Detection and analysis of low concentrations of biomolecules for medical diagnostics, environmental monitoring, and quality control of products have always been of great interest and active topic of research due to associated benefits. For instance, precise detection of cancer biomarkers in blood allows timely diagnosis and treatment of the disease or detection of airborne pathogens can lead to early counter measures. However, the isolation and detection of these biomolecules present many challenges due to the fact that biomolecules are part of complex environments, such as blood, urine, air or water, and numerous other chemical and biochemical agents. Another challenge is the nanometer size scale of the biomolecules and need for same size devices to interact with them. To meet these challenges the collaboration of various engineering and science disciplines under the umbrella of nanotechnology and nanoscience has brought together innovative methods and techniques to design smaller and better devices known as nanobiosensors or simply biosensors-devices that can identify and recognise biological molecules and their activities. Blood, gas, glucose monitoring devices and pregnancy test strips are a few examples of commercially available biosensors, which not only benefit the patients but also underscore the need and the potential for more sophisticated systems even for DNA and protein detection (Gruhl et al., 2013; Carrascosa et al., 2006; Murphy, 2006; Wang, 2006; Collings and Caruso, 1997).

*Author for correspondence; E-mail: kmarif@uet.edu.pk
Generally, a biosensor has three major units, detector, transducer and signal processor. The detector accomplishes target specific recognition to detect the biochemical phenomenon (e.g., antibody-antigen binding), the transducer (e.g., cantilever, nanowire) generates measurable signals, and finally the processor unit (e.g., microcontroller or computer) filters, amplifies, and displays the output signal. Nanotechnology has been playing a vital role to better all these units (biosensors, detectors and transducers) therefore, getting benefits of devices based on these technologies. Utilising the concepts from already advanced field of semiconductor manufacturing, researchers have started producing micro-electro-mechanical systems (MEMS) and nanoelectro-mechanical systems (NEMS) out of silicon well as polymers, metals, carbon, and etc., (Staples et al., 2006; Grayson et al., 2004; Moore and Syms, 1999). These days, some MEMS and NEMS devices are commercially available in the market (e.g., drug delivery systems, micro-needles, stents), (Murphy, 2006; Staples et al., 2006; Grayson et al., 2004) and some of them readily outperform widely used conventional systems. Their success attributes to short response time, mass fabrication, and capacity to integrate with other lab-onchip devices and undoubtedly opens up possibilities of cost effective and portable devices for detecting biomolecules (Dhayal et al., 2006; Ziegler, 2004; Arntz et al., 2003).

A variety of biomolecular detection sensors based on nanodevices have been reported in the last many years 
in the published literature. Similarly, numerous comprehensive review articles on various perspectives of nanobiosensing have also been published (Gao et al., 2012; Yeom et al., 2011; Curreli et al., 2008; Erickson et al., 2008; Nicu and Leichle, 2008; Cheng et al., 2006; Patolsky et al., 2006; Wanekaya et al., 2006). Contrary to usual reviews, main emphasis in this review was on the detectors and transducers that are direct result of nanotechnology, e.g., cantilevers, nanowires (NWs) and carbon nanotubes (CNTs). Properties and usage of these nanodevices have been discussed for particular biomolecules, however, signal transduction, processing and other aspects have been only mentioned if needed to elaborate a certain technique. A brief description of each device is followed by common sub-types of device variants and methods to incorporate in a biosensor design as detector and/or transducer. Then research examples from literature have been discussed to highlight the challenges and achievements in that particular category.

Micro cantilevers. Micro cantilevers are diving-board like structures that fall in the category of mechanical detection of biomolecules. A typical schematic diagram of the optical measurement setup, scanning electron micrograph (SEM) of micro-fabricated cantilevers and an illustration of molecular hybridisation and bending of cantilevers are shown in Fig. 1. Generally, the cantilevers are fabricated from silicon ( $\mathrm{Si}$ ), silicon oxide $\left(\mathrm{SiO}_{2}\right)$ and silicon nitride $\left(\mathrm{Si}_{3} \mathrm{~N}_{4}\right)$ through state of the art and standard semiconductor fabrication methods. However, specific applications and signal transduction methods may require coating of cantilevers with different materials to increase affinity for certain probe molecules or to provide shiny surface for laser reflection. Usually, gold $(\mathrm{Au})$ coatings are used because alkane chain with thiol groups binds to gold and proteins adsorb on gold surface (Raiteri et al., 2001; Storri et al., 1998).

Gold coated Si cantilevers arrays were used by Fritz et al. (2000), as pioneering work in the field, to detect mismatch of oligonucleotides. Later on, arrays of individually functionalised cantilevers were employed for label-free multiple DNA detection by McKendry et al. (2002) as well. These works were reported more than a decade ago and since then gold coating has widely been adopted as preferred method of immobilisation. Polymer based cantilevers for biosensing applications have also been reported. Fluorocarbon coated polymeric cantilevers have been shown to have

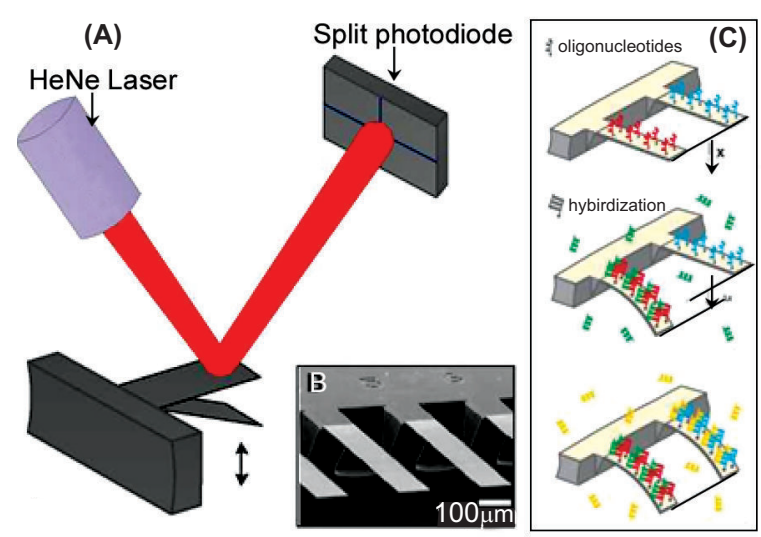

Fig. 1. (A) Schematic diagram of laser deflection setup used to measure transverse vibrations of the mechanical oscillators. (B) SEM micrograph of a section of a microfabricated Si cantilever array. (C) Scheme showing an example of hybridisation experiments with cantilever functionalisation on one side (Fritz et al., 2000).

some advantages over gold-coated silicon nitride cantilevers such as stability for temperature and $\mathrm{pH}$ changes (Calleja et al., 2006). However, the fabrication and immobilisation techniques for developing higher sensitivity polymer cantilevers require further improvements.

Cantilevers are mainly used to respond to stress or mass changes on the surface of the free end. Stress change results in deflection of the free end of the cantilever (static mode), while mass change influences both vibration frequency (resonant mode) and deflection. In static mode, the adsorption of molecules onto the surface causes the cantilever to bend. The well-known Stoney's equation (Stoney, 1909) explains the relation between the surface stress change and cantilever's tip deflection:

$$
\Delta \mathrm{z}=3 \frac{(1-\mathrm{v})}{\mathrm{E}} \frac{\mathrm{L}^{2}}{\mathrm{t}^{2}} \Delta \sigma
$$

where:

$\Delta \mathrm{z}=$ the cantilever's tip deflection; $\mathrm{v}=$ Poisson's ratio; $\mathrm{E}=$ Young's modulus; $\mathrm{L}=$ the length of the cantilever; $\mathrm{t}=$ is the thickness of the cantilever; and $\Delta \sigma=$ change in the surface stress $(\mathrm{N} / \mathrm{m})$. The Stoney's equation could be employed to estimate the tip deflection of simple rectangular (Fig. 1) or complex (Fig. 3) cantilever geometries. 
When the cantilever is loaded with additional mass, resonant frequency decreases and the additional mass can be calculated by:

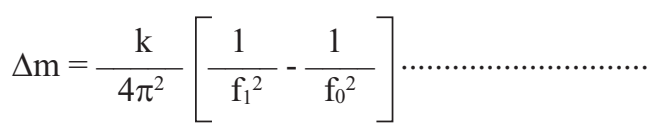

where:

$\mathrm{k}=$ the spring constant; $\mathrm{f}_{0}=$ the initial frequency; $\mathrm{f}_{1}=$ the resonant frequency after the mass loading. In order to increase the sensitivity, external actuation through piezoelectric devices may be used to create vibrations at specific frequencies (Johnson et al., 2006; Gupta et al., 2004).

Through optical and/or electrical methods, changes in tip deflection and resonant frequency may be readily detected and amplified for correlation (Johnson et al., 2006; Sone et al., 2006; Wu et al., 2001; Fritz et al., 2000; Ilic et al., 2000). However, laser based measurements are considered superior to other methods due to their higher sensitivity. Figure 1 shows a laser based measurement setup (also called optical lever method) wherein a laser beam, reflected from the surface of the cantilever, is detected by split photo-detectors that can measure the deflections with high accuracy $(0.1 \mathrm{~nm})$ as reported by Fritz et al. (2000).

Gupta et al. (2004) demonstrated detection of Listeria innocua cells using micro-fabricated cantilevers in resonant mode (Fig. 2). In this work, the cantilevers were loaded with cells and frequency shifts were observed on an optical measurement setup. It was observed that 180 bacterial cells caused nearly $2 \mathrm{kHz}$ frequency shift. This work not only highlighted the modes of operation of cantilevers for biosensing but also served as seminal preliminary work for further development. In the recent years, numerous biosensing systems based on surface functionalised cantileversthrough immobilising biomolecular probes-have been reported for detection of various biological targets such as glucose (Pei et al., 2004), bacterial cells Escherichia coli (Gfeller et al., 2005), Bacillus subtilis spores (Dhayal et al., 2006), Vaccinia virus particles (Johnson et al., 2006), RNA (Zhang et al., 2006), and liposomeprotein interaction (Hyun et al., 2006).

Cantilevers with shape novelties. Even though standard mechanical shape of a cantilever has one fixed and the other free end (Fig. 1), various geometrical changes are possible to meet the particular requirements of signal transduction and surface biochemical reactions. In this
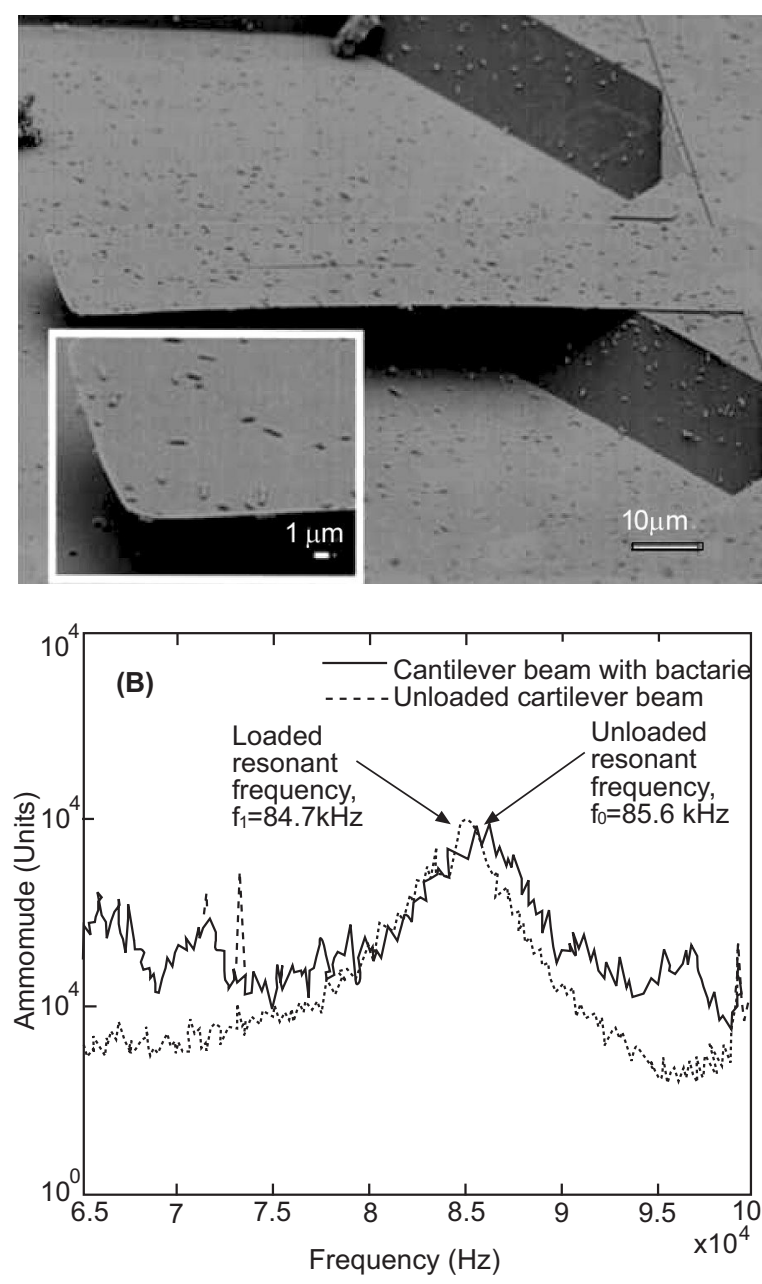

PSD of cantilever 1 after unloaded lever binding of around 180 bacterial cells

Fig. 2. (A) SEM micrograph of Listeria innocua cells on the cantilever surface. (B) Unloaded and loaded resonant frequencies of the cantilever (Gupta et al., 2004).

regard, fabrication of cantilevers for biosensor design with integrated optical interferometric features has been a hot topic of research for many years. Originally, these cantilevers were developed for atomic force microscopy (AFM), where cantilevers were used as scanning probes (Onaran et al., 2006; Yaralioglu et al., 1998; Manalis et al., 1996).

The first ever cantilever biosensor incorporating interferometry features was reported by Sulchek et al. (2001). In this work, the transducer, with interdigital fingers at the end of the cantilever to form a diffraction grating, was fabricated from $\mathrm{Si}_{3} \mathrm{~N}_{4}$ and coated with $20 \mathrm{~nm} \mathrm{Au}$ by evaporation. During experiments, hydrophobic 
perfluorocarbon was applied to one arm (reference) to prevent covalent binding between the cantilever surface and thiol groups. Later, the thiol self-assembled monolayers (SAMs) on the other cantilever (sensor) were observed with optical lever and interferometry methods. It was found that interferometry method produced signalto-noise ratio (SNR) of 18, while optical lever method resulted in SNR of 3.

The sensor shown in Fig. 3 (Savran et al., 2004) was designed to sense surface stress changes caused by the adsorption of biomolecules onto the cantilever. In this example, the molecules of interest were aptamers [single-stranded DNA or RNA (ssDNA or ssRNA) molecules that bind with high affinity and specificity to proteins and peptides etc.] and ligands. Aptamer molecules were immobilised on the gold-coated sensor surface with a thiol linker. ssDNA was immobilised to the reference cantilever in order to prevent nonspecific binding of target molecules to the reference cantilever. The L-shaped geometry of the sensor (Fig. 3A) allowed each cantilever to be functionalised individually by dipping one side (either sensor part or reference part) into a micropipette. In the experiment, ligand-aptamer binding created a surface stress change, which bent the sensor cantilever, while the reference cantilever was unaffected. The tip deflection of the cantilever was measured by a laser beam based on interferometry. Injection of $500 \rho \mathrm{M}$ Taq DNA polymerase solution into the chamber, including the cantilever, caused a differential deflection of $32 \mathrm{~nm}$, while injection of $75 \mathrm{nM}$ thrombin solution did not induce a measurable differential deflection. These results proved that differential deflection occurred as a result of specific binding.

Differential measurements with interferometry have considerable advantages over single measurements in terms of background noise. However, researchers who do not use differential cantilevers employ other methods to reduce noise. For instance, Fritz et al. (2000) employed one cantilever as a control and used optical lever measurements twice to subtract background noise from the biological signal. In another case, Alvarez and Tamayo (2005) employed a scanning laser source for recording measurements from cantilever arrays. Both of these techniques required additional signal processing and more complex measurement systems, when compared with the interdigitated cantilevers.

Cantilever geometries are not necessarily modified for sensitive measurements or noise reduction as mentioned
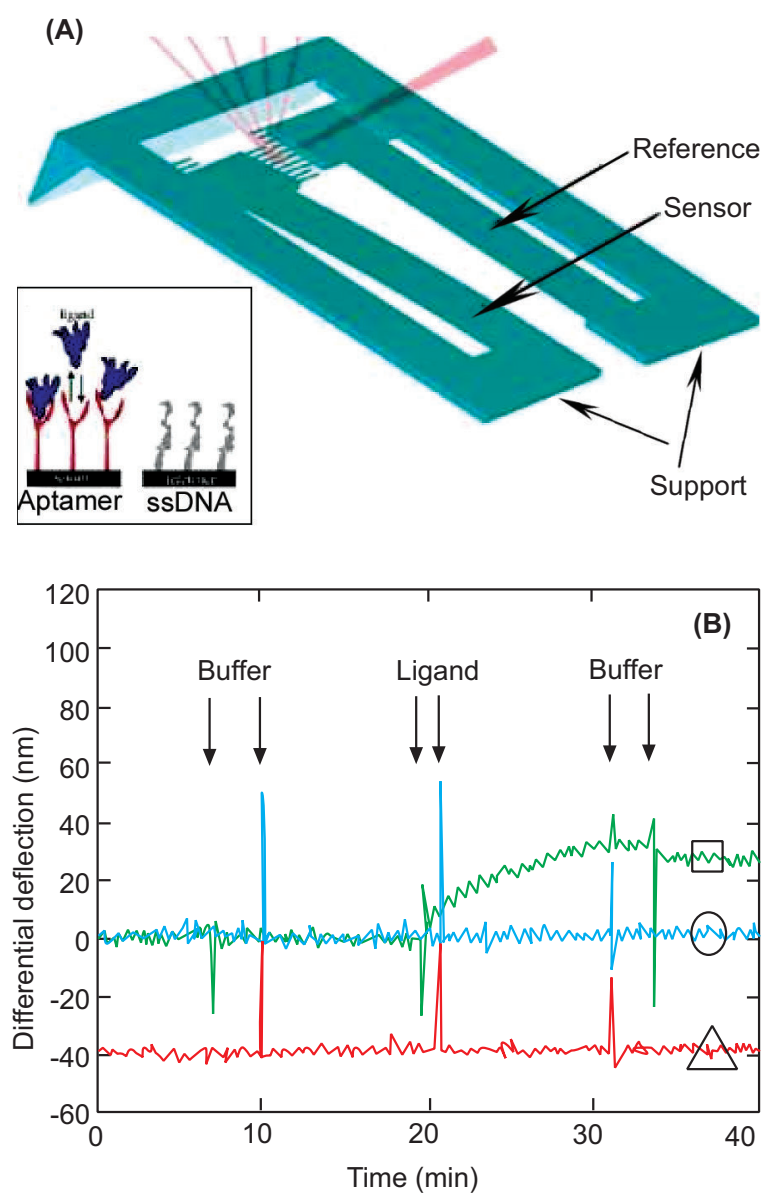

Fig. 3. (A) Schematic layout of an interferometric cantilever sensor. Both cantilevers (sensor and reference) are supported by L-shaped thick structures. Inset shows configuration of the front view of Au coated and functionalised sensor and reference cantilevers. (B) The result of DNA polymerase ( $\square$ ) and trombin $(O)$ injection $(\Delta)$ is the response to Taq DNA polymerase when both the sensor and the reference are functionalized with ssDNA (Savran et al., 2004).

above. Fabrication of microfluidic channels on the top surface of cantilevers (Fig. 4A) was introduced by Burg et al. (2007) and Godin et al. (2007) and since then numerous devices with embedded microfluidic channels have been reported (Grover et al., 2011; Park et al., 2010). Burg et al. (2007) produced a microfluidic channel on the top surface of Si cantilever to weigh cells, biomolecules or particles by delivering them to the tip of cantilever through a continuous flow of fluid. The device was reported to have lower resolution $\left(0.01 \mathrm{ng} / \mathrm{cm}^{2}\right)$ compared to quartz crystal microbalance 
(QCM) and surface plasmon resonance (SPR). The device was used for both bound and unbound masses (Fig. 4B), however, the bound species paved way for specific detection by way of immobilisation. Experiments with particle position dependent signal measurements were also conducted. In these cases, the peak frequency shifts induced at the apex quantified the exact mass excess of a particle (Fig. 4C).

(A)

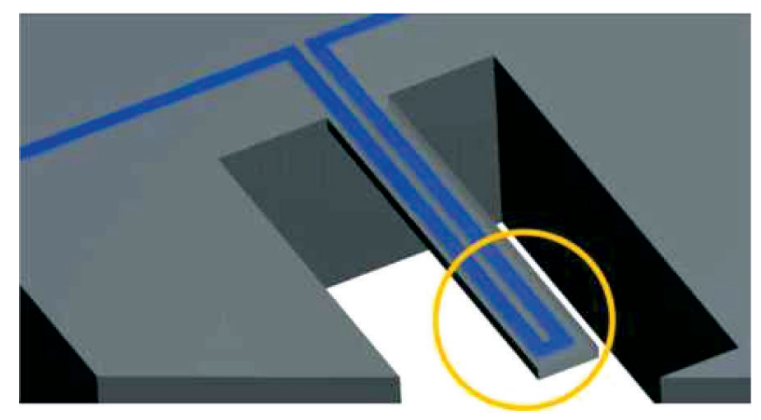

(B)

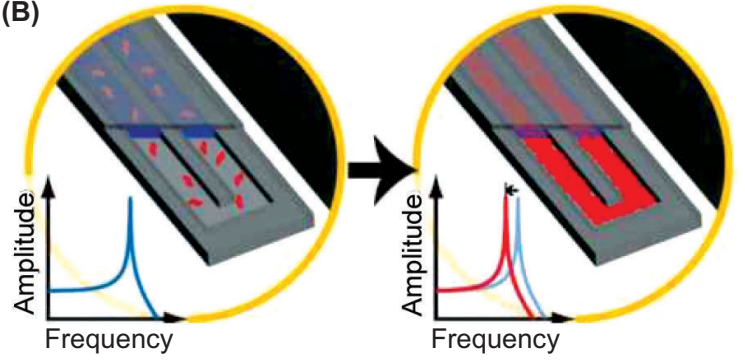

(C)

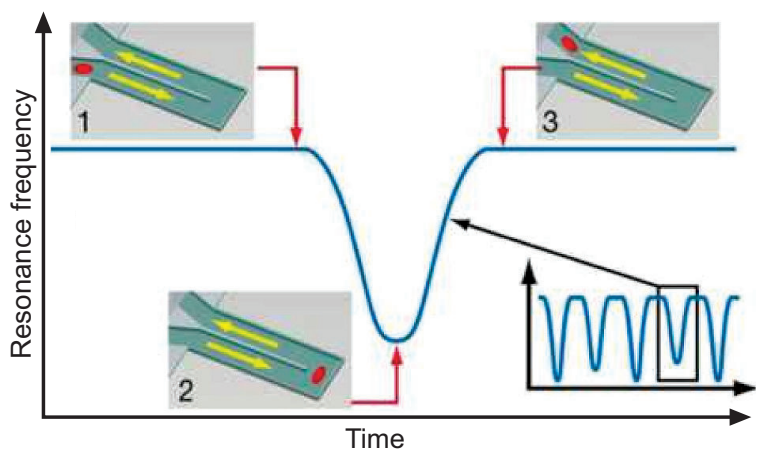

Fig. 4. (A) Schematic illustration of a cantilever with buried microfluidic channel to allow two mass measurement modes. (B) Frequency shift for accumulated (due to binding to the channel wall) particles shows possibility of specific detection by way of immobilised receptors. (C) Signal dependence on the position of flowing particle (inset 1-3) with the peak frequency shift induced at the apex (Burg et al., 2007).
Cantilevers with magnetic nanoparticles. According to equation 2 , the sensitivity of measurements with cantilevers depends upon frequency of vibration, which in turn depends on the mass of particle. However, the loaded particles (biomolecules, cells, viruses, etc.) do not generally produce large frequency shifts e.g., a frequency shift of only $2 \mathrm{kHz}$ was observed by Gupta et al. (2004). One of the methods to increase mass is by using magnetic nanoparticles (or beads) with cantilevers. For interaction with biomolecules, the surface of these nanoparticles can be modified by attaching the appropriate ligands such as antibodies, proteins or oligonucleotides (Bryant et al., 2007).

Moreover, these particles are magnetic only under the magnetic field, they could be easily separated from complex environments by applying external magnetic fields (Neuberger et al., 2005). A demonstration of the employment of magnetic beads with cantilevers for biosensor application is illustrated in Fig. 5 (Weizmann et al., 2004).

The setup shown in Fig. 5 was used for ultra-sensitive detection of viral (M13 $\varphi$ ) DNA, single-base mismatch in a nucleic acid, and telomerase (Weizmann et al., 2004). Si cantilevers were coated with Au and functionalised with avidin and the deflection of cantilevers was measured by laser beam reflection on a photodiode, using an optical setup similar to Fig. 1A. Functionalised magnetic beads were injected onto the cantilevers to bind with avidin already present on the cantilever surface. When a magnetic field was introduced by an external magnet, the deflection of the cantilever was observed and $7.1 \times 10^{-20} \mathrm{M}$ of M13 $\varphi$ DNA was reported as the minimum detectable concentration with this method.

For the single-base mismatch (mutant gene) experiment the same procedures were employed. Functionalised magnetic beads (appropriately probed) hybridised with normal genes resulted in cantilever deflection, whereas, no signal change was observed in the presence of the mutant gene. Similarly, for the telomerase experiment, functionalised magnetic beads were introduced to HeLa cancer cell extract in the presence of telomerase, nucleotides, and biotinylated dUTPs (deoxyuridine triphosphate). When the magnetic beads coupled with biotin label and telomere bound to the avidin-coated cantilevers, the applied magnetic field caused deflection. A detection limit of 100 cancer cells was reported. 
(A)

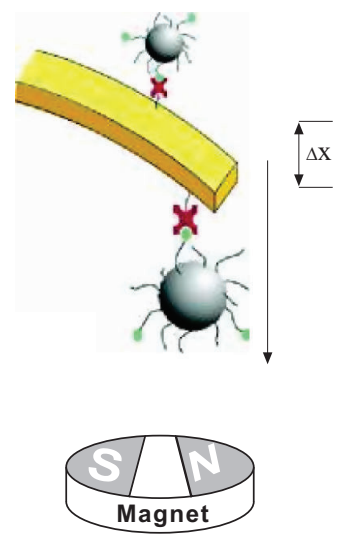

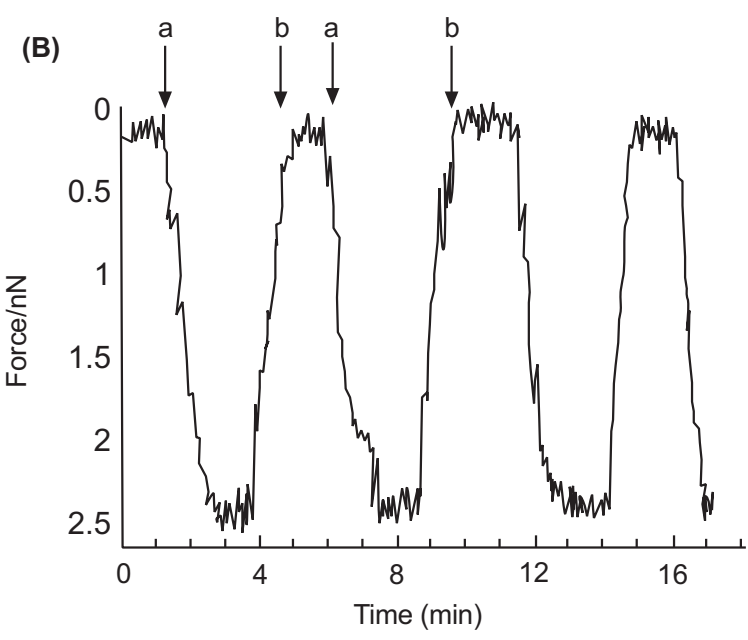

Fig. 5. (A) Schematic layout of magneto-mechanical analysis of biorecognition process on functionalised cantilevers. (B) Magneto-mechanical deflection of the cantilever. At points marked as 'a' cantilever is subjected to the external magnet, while at points marked as ' $b$ ' the external magnet is removed. Adapted from Weizmann et al. (2004).

Another biosensor application of magnetic beads and cantilevers for detection of endonuclease activity has been presented by Weizmann et al. (2005). Gold-coated cantilevers were functionalised with nucleic acids and the magnetic beads were functionalised with complementary nucleic acids. The endonuclease activity was tested by DNA cleavage and detected by cantilever deflection when magnetic field was applied.

Furthermore, analysis of actuation setup for the two systems in Weizmann et al. (2005) and Weizmann et al. (2004), tells that sensitivity of cantilevers with standard rectangular geometries could be increased by magnetic beads, when a permanent magnet is placed underneath to apply force to cantilevers. Weak magnetic field intensity causes no deflection, whereas when the magnet is moved towards the cantilever, stronger magnetic field intensity causes bigger deflections. However, the noise in the output signal remained as a limiting factor and these sensors only served as early stage development in the area of force applied biological sensor.

Building upon findings of interferometric (Savran et al., 2004) and magneto-mechanical (Weizmann et al., 2005; Weizmann et al., 2004) cantilever devices, recently Icoz et al. (2008) presented actuation of interferometric cantilevers at low noise region using magnetic beads as shown in Fig. 6. The control arm of the differential cantilever was passivated with bovine serum albumin (BSA) and sensing arm was probed with biotin-BSA. The probe molecules were placed on the cantilevers using nanojet dispensing system as an alternative method to micro-pipetting (Bietsch et al., 2004). Nanoject dispensing was reported to have allowed only one side of the cantilever to interact with the biomolecules and only small amount of drops were needed to functionalise the surface. The electromagnet was controlled by a function generator so that the frequency of excitation signal could be adjusted. In this way, cantilevers were excited at low noise region allowing a resolution of $0.065 \AA$. Later, using this setup, $0.28 \mathrm{nM}$ concentration of streptavidin was detected (Icoz and Savran, 2010). In this study, streptavidin from serum was captured and separated by biotin-coated magnetic beads.

Nanowires. Nanowires (NWs) are nanometer sized structures with large aspect ratios. This small size and large aspect ratio renders them very different physical and electrical properties compared to bulk material from which they are made e.g., very small conductivity due to scattering from boundaries. NWs fabricated out of $\mathrm{Si}$ and indium oxide (Ahn et al., 2012; Li et al., 2005; Tang et al., 2005) are commonly used in biosensor applications. Both, top-down (Ahn et al., 2010) and bottom-up (Zheng et al., 2005) methods are employed 

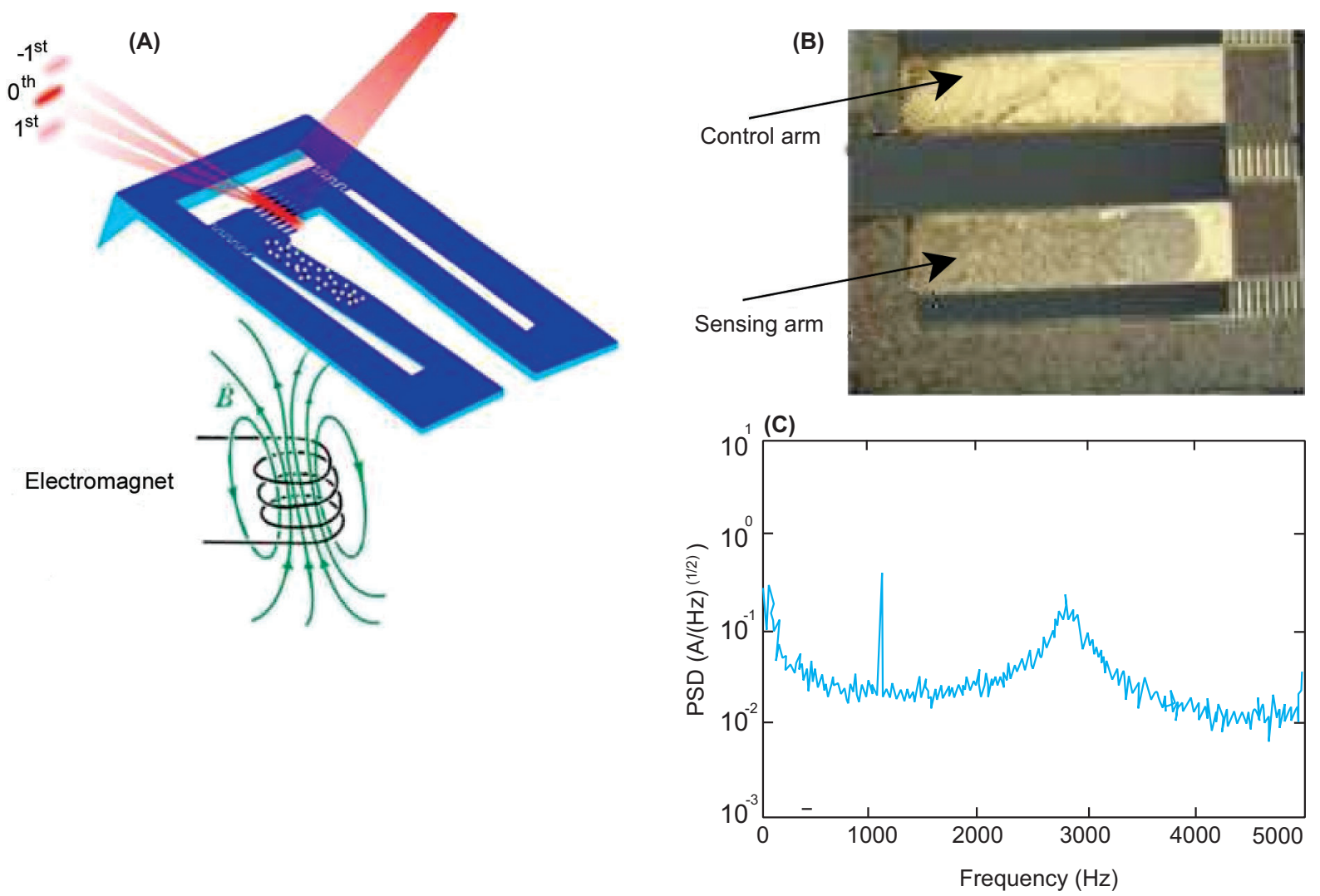

Fig. 6. (A) Magnetic actuation and interferometric detection scheme. (B) Magnified image of the functionalised cantilever device showing insignificant number of magnetic beads on the control arm and huge numbers of the same bound to the sensing arm. (C) Cantilever actuation at $1103 \mathrm{~Hz}$ and resonant frequency around $3000 \mathrm{~Hz}$ (Icoz et al., 2008).

for NW fabrications. Top- down methods, which utilise lithography, deposition, and etching, randomly orient the NWs and they need to be positioned and aligned on the electrodes through different alignment techniques (He et al., 2008). Bottom-up methods, which utilise chemical vapour deposition (CVD), are better for functionalisation before the alignment on the chip and hence easier than functionalising multiplexed NWs already placed on a chip. NWs which are lightly doped, have short length and smaller diameter, show increased sensitivity. However, in order to have smaller diameters new fabrication techniques have to be developed (Stern et al., 2008).

Mostly, NWs are used in biosensors as NW field effect transistor (NW-FET) and their structure is similar to conventional FETs, which includes drain, source, semiconductor channel, and gate electrodes. NW forms the semiconductor channel with a high surface areato-volume ratio. The binding of biomolecules to the
NW causes accumulation or depletion of charge carriers both on the wire surface and inside the wire resulting in detectable conductivity changes. Detection of antibodies (Stern et al., 2007), ssDNA (Kim et al., 2007), virus (Patolsky et al., 2004), proteins (Cui et al., 2001), and electrical activities of neuron cells (Patolsky et al., 2006) have been reported. A typical representation of these FETs is given in Fig. 7.

Use of NWs is not limited to FET-based sensors, instead they have been used in many other ways such as amperometric (Lu et al., 2007), impedimetric, and potentiometric (Zhang et al., 2009). However, for the sake of brevity, NW-FET-based sensors have been discussed here. An elaborative investigation of such electrochemical biosensors has been done by Grieshaber et al. (2008).

In case of NW-FETs, the effective magnitude of surface charge highly depends on the ionic concentration and ionic strength of the buffer solution. Ionic strength (I) 
(A)

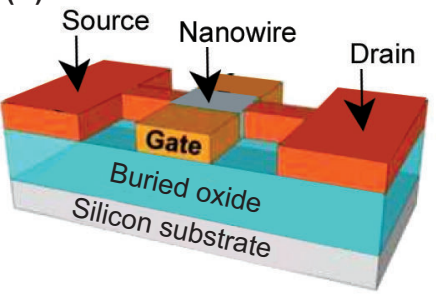

(B)

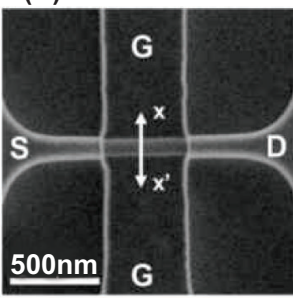

Fig. 7. (A) Illustration of a nanowire FET biosensors fabricated on Si substrate. NW surface is functionalised with receptor molecules to capture target molecule. (B) SEM micrograph of the top view of the fabricated biosensor showing Source, Drain and Gate of FET (Ahn et al., 2012).

of the buffer solution determines an important parameter, the Debye length the length in which mobile charge carriers screen out the external electric field, causing small charge movement (Fig. 8), given by Maehashi et al. (2007).

$$
\text { Debye length } \sim 0.32(\mathrm{I})^{-12} \text {. }
$$

In order to improve device performance against Debye length limitation (equation 3), various probe molecules have been investigated. Advancement in probe molecules is linked with the advancement in the NW based biosensors.

Nanowires and probe molecules. Functionalisation of Si NW-FETs with aptamers (1-2 nm), oligonucleic acid or peptide molecules that bind to a specific target molecule, helps reduce the Debye length as compared to antibodies $(\sim 10 \mathrm{~nm})$ (Fig. 8). This strategy has been used to detect thrombin (Kim et al., with $\sim 330 \mathrm{\rho mol} / \mathrm{L}$ sensitivity and to detect vascular endothelial growth

(A)

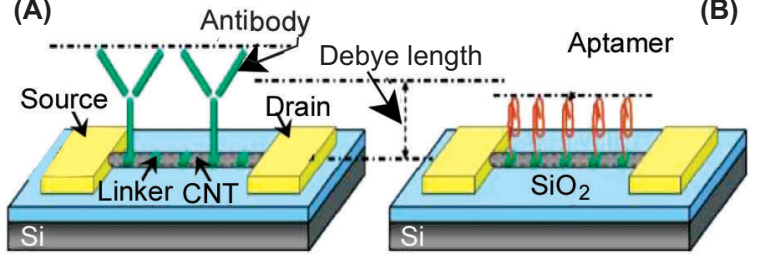

Fig. 8. Illustration of Debye length in NW/CNTFET biosensor. (A) Functionalisation with antibody results in size larger than Debye length, while (B) aptamer functionalisation produces size smaller than Debye length and thus causes higher charge interaction (Maehashi et al., 2007). factor (VEGF) with $100 \rho \mathrm{M}$ sensitivity (Lee et al., 2009). Sophisticated probe molecules can be designed through protein engineering, leading to detection of uncharged hormones (steroid, 19- noradrostendione) with Si-FETs (Chang et al., 2009). The reported sensitivity in this case is in $\mathrm{fM}$ range. Engineered antibody mimic proteins (AMPs) have improved binding affinity and recognition with sizes (typically 2-5 nm) smaller than antibodies (Ishikawa et al., 2009). The application of AMPs on indium oxide FETs was demonstrated to detect SARS biomarker N-protein. When compared to enzyme-linked immunosorbent assay (ELISA) which takes hours, this method only takes $10 \mathrm{~min}$ to detect SARS biomarker.

Immobilising peptide nucleic acid (PNA) on the nanowires does not cause significant charge formation on the surface thus, yielding higher sensitivity. The hybridisation efficiency of PNA and micro RNA (miRNA) have been shown to be higher than DNA and miRNA (Zhang et al., 2009). In this study, PNA functionalised Si FET sensor was able to detect $1 \mathrm{fM}$ of miRNA. PNA functionalised $\mathrm{Au}$ NWs were used to detect $100 \mathrm{fM}$ of mRNA (Fang and Kelley, 2009), while a previous study reported PNA functionalised Si FET sensor to detect $10 \mathrm{fM}$ of DNA (Gao et al., 2007). One method of covalent binding of PNA molecules to $\mathrm{SiO}_{2} \mathrm{NW}$ has been described by Cattani-Scholz et al. (2008), which is an alternative way to physisorption. Zhang et al. (2010) recently reported a highly sensitive and rapid sensor based on PNA-DNA hybridisation to detect reversetranscription-polymerase chain reaction (RT-PCR) product of Dengue serotype 2 (DEN-2). As shown in (Fig. 9), Si NW was functionalised with PNA and resistance change of the sensor before and after hybridisation was measured. Compared to previously reported long hybridisation time (about $16 \mathrm{~h}$ ), this scheme worked for as little as $30 \mathrm{~min}$ of hybridisation and showed $6 \%$ response change, which corresponds to detection of $10 \mathrm{fM}$ concentration of the RT-PCR product of DEN-2. The assay is also reported as highly reproducible, with less than $15 \%$ relative standard deviation on different chips.

NWs may also be functionalised with lipid bilayers (Martinez et al., 2009). The lipid bilayers include various types of ion channels, so these channels can act as barrier and transport the specific ions through NW. In this proof-of-concept work, lipid membrane with $1.4 \mathrm{~nm}$ pores formed by $\alpha$-hemolysin polypeptide promoted the ion transport. This kind of selectivity can 
A
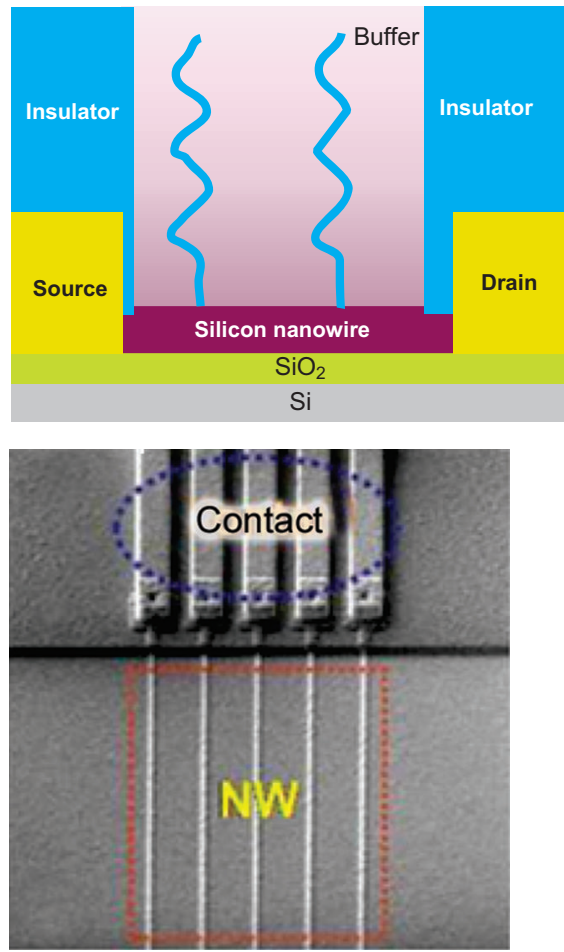
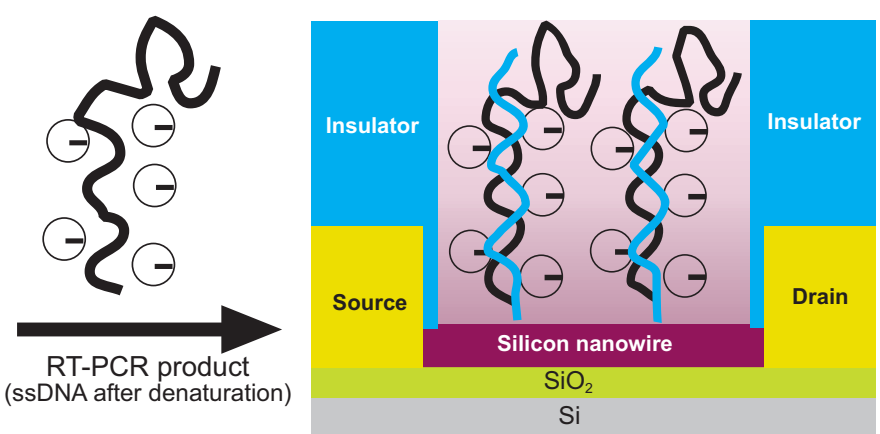

$\mathrm{Si}$

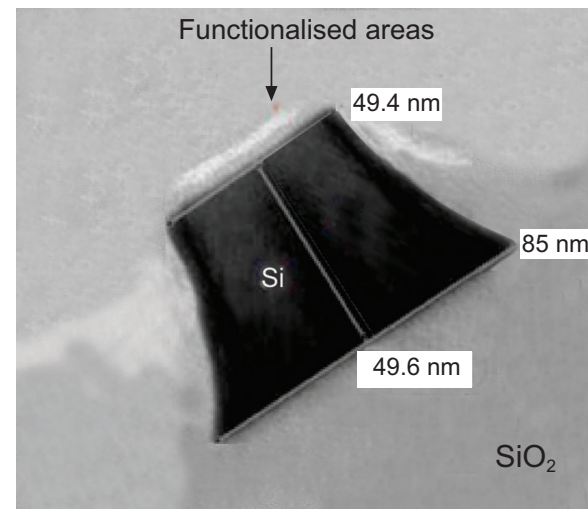

Fig. 9. Schematic diagram of the RT-PCR product of DEN-2 hybridised to the PNA-functionalised Si NW sensor. (B) SEM image of NW arrays and the corresponding contact lines. (C) TEM image of a single NW embedded in $\mathrm{SiO}_{2}$ after surface functionalization (Zhang et al., 2010).

shield the NW surface from the other ions present in the surrounding liquid.

Novel materials for nanowires. In order to reduce the fabrication costs and overcome the limitations of NW reproducibility, and non-specific binding of biomolecules, other materials such as polymers and hybrid materials are also being investigated. Polymers draw attention because of their electrical, magnetic and optical properties. Electrochemically synthesised polypyrrole (Ppy) - a conducting polymer-NW has been used to make a chemiresistive biosensor to detect cancer marker, CA 125 , where $1 \mathrm{U} / \mathrm{mL}$ was reached as the limit of detection (Bangar et al., 2009). Another work on conducting polymer (pyrrolepropylic acid) FET resulted in $50 \mathrm{nM}$ detection limit for human serum albumin (Tolani et al., 2009). Electrochemical detection of oligonucleotides has been demonstrated by using polyaniline nanotubes with low nanomolar detection limit (Zhang et al., 2007). In an electrochemical impedance spectroscopy experiment, GaN NWs with diameter of 25-100 nm were fabricated with CVD process for the detection of DNA (Chen et al., 2009). For a FET sensor, poly-Si NWs with diameters of $80 \mathrm{~nm}$ were fabricated applying a low-cost sidewall spacer technique, where fM DNA detection limit was reported (Lin et al., 2009). Another material investigated for NW biosensor is iridium oxide $\left(\mathrm{IrO}_{2}\right)$ (Zhang et al., 2008). $\mathrm{IrO}_{2}$ has been reported to have advantages when used as a stimulating and recording electrode and characterised NW has been proposed for biosensing or neuron electrode.

A multi-segment NW-FET biosensor was introduced for ssDNA detection (Wang and Ozkan, 2008). In this study, NWs had a heterostructure of CdTe-Au-CdTe with $230 \mathrm{~nm}$ diameters. Electrochemically deposited $\mathrm{CdTe}$ and $\mathrm{Au}$ NWs had a $p$-type semiconductor behaviour. Au part of the NW was functionalised with thiolssDNA and $1 \mu \mathrm{M}$ of ssDNA detection was reported. Even though the sensitivity is not at the desired level, multisegment NWs have the advantage of selective functionalisation. Thus, the non-specific binding of probe molecules to electrode contacts and bulk material can be avoided.

Instead of using single wire, using multiple wires can be advantageous in some applications. For example, detection of bacteria based on impedance measurements 
are reported by Wang et al. (2008), where $\mathrm{TiO}_{2} \mathrm{NW}$ bundle ( $\sim 1 \mathrm{~mm}$ length) was easily placed on electrodes under optical microscopy. In another example, Si NWs with $30 \mathrm{~nm}$ diameters and $100 \mu \mathrm{m}$ length were fabricated using a top-down approach. As it was mentioned earlier, top-down approach leads to highly controlled orientation of NWs. The orientation and size of the NWs allowed detection of electrical activity of cardiac cells (Pui et al., 2009).

Carbon nanotubes. Carbon nanotubes (CNTs) are very thin (with diameter in $\mathrm{nm}$ ), hollow cylindrical structures made of carbon atoms. CNTs can be mainly classified into single-wall carbon nanotubes (SWCNTs) or multiwalled carbon nanotubes (MWCNTs) depending upon the location of carbon atoms on the surface of nanotubes. Figure 10 depicts three different types of SWCNTs resulting from roll-up of graphene sheet and a MWCNT made up of three shells of differing chirality (a property of asymmetry). When CNTs are fabricated by CVD the semiconducting properties are controlled during the growth process. Generally, CNT biosensors consist of either SWCNTs or a network of nanotubes (Claussen et al., 2009). SWCNTs demonstrate ultra-high sensitivity because of their size ( $\sim \mathrm{nm}$ diameter). When using CNTs in FETs they form the semiconductor channel and interact with the analyte in a similar fashion as nanowires (Fig. 7-8).
(A)

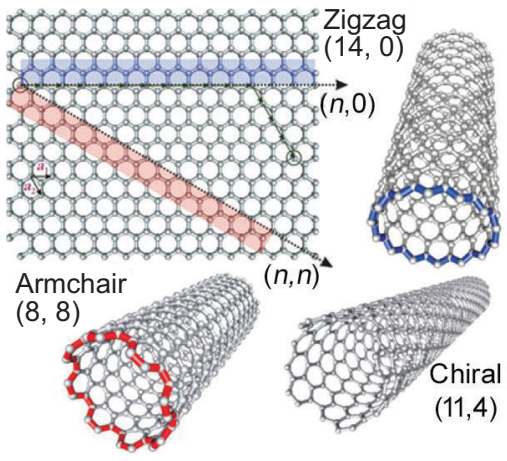

(B)

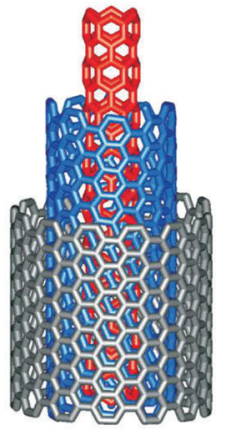

Fig. 10. (A) Three different types of SWCNTs result from roll-up of graphene sheet. Numbers in parentheses, $\mathrm{m}$ and $\mathrm{n}$, are positive integers called chiral indices. These two numbers govern all physical properties of SWNTs. (B) Structure of a MWCNT made up of 3 shells of differing chirality (Balasubramanian and Burghard, 2005).
Proteins (Strehlitz et al., 2008; Kojima et al., 2005), glucose (Besteman et al., 2003), DNA (Li et al., 2003) and swine influenza virus H1N1 (Lee et al., 2011) are among a few demonstrations of CNT-FETs in biosensing applications. CNTs could also be designed as part of capacitive biosensors e.g., SWCNT network formed the one plate of the capacitor in a sensor designed for the detection of prostate specific antigen (PSA) (Briman et al., 2007). The CNT network was functionalised with specific antibody against PSA. The charge alteration was detected by measuring the capacitance change of the circuit and a detection limit of $100 \mathrm{ng} / \mathrm{mL}$ was reached in untreated calf blood serum, although in previous works with NWs (Li et al., 2005; Zheng et al., 2005) lower detection limits of PSA were reported.

Numerous valuable review articles have been written on CNT-based sensors (Musameh et al., 2012; Kerman, 2008; Allen et al., 2007; Gruner, 2006), therefore, in this part, present discussion is confined to most recent research and progress directed towards improvement in sensing capabilities of CNT-FET biosensor only.

Though single molecule level detection schemes using CNT-FETs have been demonstrated (Choi et al., 2012; Sorgenfrei et al., 2011), problems such as sensor-tosensor variation, non-specific binding and charge noise are among major issues associated with CNT-FET biosensors. Recently, determination of extent and cause of these problems has gotten attention of many researchers. A dual-mode biosensor was reported by $\mathrm{Oh}$ et al. (2010), wherein CNT-based metal semiconductor FET (CNT-MESFET) structure was fabricated on a quartz substrate (Fig. 11). The Au strip (middle of the CNT channel) acted as the top gate of FET because of the Schottky contact between the CNT and the Au strip. DNA hybridisation occurring on the Au top gate can be detected by simultaneously measuring the change in the electrical conductance and the SPR. These two techniques were chosen, in an expectation, for this dualmode biosensor to provide high sensitivity and reliability in electrical and SPR measurements, respectively. The authors report a shift in SPR reflectance minimum and decrease in the $I_{S D}$ (current between source and drain), upon hybridisation of DNA with the Au top gate. It was further noticed that SPR measurements could be reproduced independent of the sensor and thus could be used to measure sensor-to-sensor variation of conductance. 


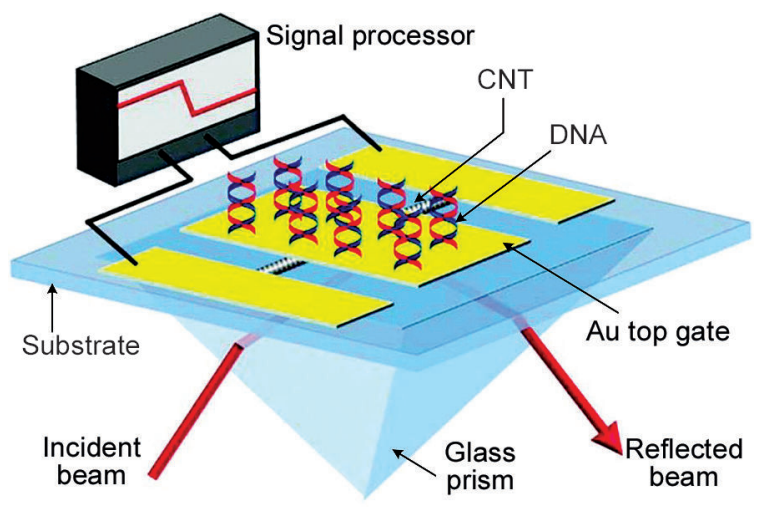

Fig. 11. Layout of a dual-mode CNT-MESFET biosensor. Adapted from Oh et al. (2010).

To better understand the mechanism and origins of charge noise in CNT-based FETs, a noise measurement setup have been reported by Sharf et al. (2012) to bring to light new design considerations for nano-FETs that are used to interface biological systems with electronics (Fig. 12). The environment in contact with ultraclean
CNT was systematically controlled to quantify the contribution of noise from substrate interactions and surface adsorbates. Prior to experiments, Raman spectroscopy and transistor curves were used to quantify any lattice defects in the CNTs or measurement hysteresis in ambient conditions (Fig. 12C-12D). Identification of single CNTs was done using scanning photocurrent microscopy (inset of Fig. 12D). Initial experiment on device-to-device variability revealed that suspended CNT devices were considerably quieter than standard CNT-FET sensors. Therefore, the suspended CNT biosensor platform could be used to search for the noise sources in traditional CNT-FET sensors. It was found that the contact with substrates and adsorbates significantly, increased the charge noise in CNT-FET biosensors. For a $1 \mu \mathrm{m}$ channel length, and a measurement bandwidth of 0.1-100 Hz, the effective gate voltage fluctuations were approximately $0.5 \mathrm{mV}$ (pristine suspended), $1.1 \mathrm{mV}$ [with poly(L-lysine) or horse heart cytochrome c], $1.8 \mathrm{mV}$ (with substrate interactions), and $2.3 \mathrm{mV}$ (with substrate interactions and PR residue). The authors speculated that the fluctuating protonation state of chemical moieties near the CNT can account for this noise.

(A)
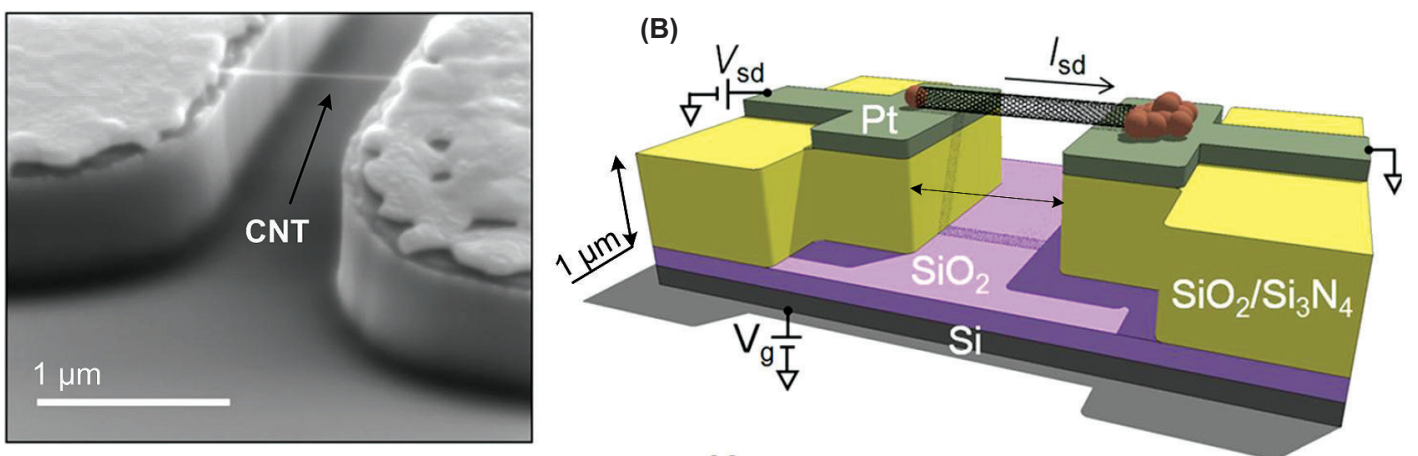

(C)

(D)
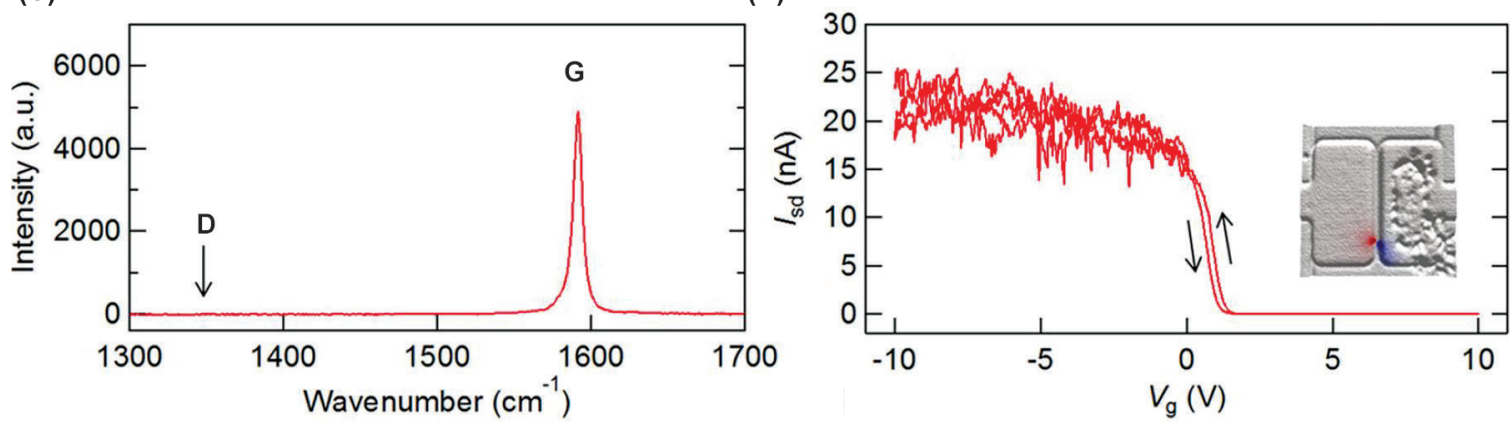

Fig. 12. (A) SEM image of a CNT bridging the gap between two platinum electrodes. (B) Schematic of hanging CNT device. (C) Raman spectra from a hanging CNT grown by fast-heat CVD. (D) Transistor curve of a hanging device in air under ambient conditions and no hysteresis. Photocurrent response (coloured dots in the inset) is superposed on top of reflectance image (Sharf et al., 2012). 
Optimisation of the sensitivity of CNT-based biosensor with biasing and surface charge is another very recent research effort (Shoorideh and Chi On, 2012) that focused on Debye screening. This work concluded that sensitivity may also be affected by other charges present in the vicinity of the analyte.

\section{Conclusion}

In this paper some emerging nanobiosensors namely cantilevers, NWs and CNTs have been reviewed. In general, advantages of these NEMS and MEMS biosensors over today's widely used procedures like ELISA, QCM, electron microscopy, and fluorescence staining assays, are short response time, mass fabrication, multiplexing and capacity to integrate with other labon-a-chip devices. They also offer the possibility of cost-effective and portable devices for detecting biomolecules. These systems have promising features, however, there are some limitations which require more research efforts.

Cantilevers have ultra-high sensitivity, however, they have not been fully developed for clinical applications. Noise and sample preparation issues need new approaches. The highest sensitivities are achieved when optical methods are used and optical methods need precise alignment with extra components. Incorporating cantilevers with magnetic nanoparticles and fabrication of microfluidic channels inside the cantilever are two different approaches to improve the cantilever operation.

NW biosensors have important features like label-free detection, ultra-high sensitivity and possibility of integration with electronic devices. Additionally, NW sensors do not need any optical components for the detection of output signals leading to more compact biosensor designs. New fabrication strategies and appropriate functionalisation methods could result in promising detection limits. As explained in the section 3, Debye length is an important limiting factor, however, alternative and novel probing agents have been developed to deal with this limitation.

CNTs are among highly investigated nanodevices researched for biosensing applications. Quite a number of recent research efforts have been directed towards understanding of noise, sensor-to-sensor variation and other problems. However, more research efforts and development are needed to optimise and understand the exact mechanism of CNT-based FET biosensors.
Overall, the influence of nanotechnology based devices on biosensors has been tremendous in the past decade or so. Many new and incremental solutions, based upon the work of other researchers, have been reported. However, there is still a long way to go for wide-spread availability and usage of these biosensors. Particularly, this is because of the fact that most of the work is still targeted towards new assays and improvements in the existing solutions. Point-of-care or clinical setting solutions are yet to come out of laboratory controlled environments.

\section{References}

Ahn, J.H., Kim, J.Y., Choi, K., Moon, D.I., Kim, C.H., Seol, M.L., Park, T.J., Lee, S.Y., Choi, Y.K. 2012. Nanowire FET biosensors on a bulk silicon substrate. IEEE Transactions on Electron Devices, 59: 2243-2249.

Ahn, J.H., Choi, S.J., Han, J.W., Park, T.J., Lee, S.Y., Choi, Y.K. 2010. Double-gate nanowire field effect transistor for a biosensor. Nano Letters, 10: 29342938.

Allen, B.L., Kichambare, P.D., Star, A. 2007. Carbon nanotube field-effect-transistor-based biosensors. Advanced Materials, 19: 1439-1451.

Alvarez, M., Tamayo, J. 2005. Optical sequential readout of microcantilever arrays for biological detection. Sensors and Actuators B:Chemical, 106: 687-690.

Arntz, Y., Seelig, J.D., Lang, H.P., Zhang, J., Hunziker, P., Ramseyer, J.P., Meyer, E., Hegner, M., Gerber, C. 2003. Label-free protein assay based on a nanomechanical cantilever array. Nanotechnology, 14: 86-90.

Balasubramanian, K., Burghard, M. 2005. Chemically functionalized carbon nanotubes. Small, 1: 180-192.

Bangar, M.A., Shirale, D.J., Chen, W., Myung, N.V., Mulchandani, A. 2009. Single conducting polymer nanowire chemiresistive label-free immunosensor for cancer biomarker. Analytical Chemistry, 81: 2168-2175.

Besteman, K., Lee, J.O., Wiertz, F.G.M., Heering, H.A., Dekker, C. 2003. Enzyme-coated carbon nanotubes as single-molecule biosensors. Nano Letters, 3: 727-730.

Bietsch, A., Zhang, J.Y., Hegner, M., Lang, H.P., Gerber, C. 2004. Rapid functionalization of cantilever array sensors by inkjet printing. Nanotechnology, 15: 873-880.

Briman, M., Artukovic, E., Zhang, L., Chia, D., 
Goodglick, L., Gruner, G. 2007. Direct electronic detection of prostate-specific antigen in serum. Small, 3: 758-762.

Bryant, H.C., Sergatskov, D.A., Lovato, D., Adolphi, N.L., Larson, R.S., Flynn, E.R. 2007. Magnetic needles and superparamagnetic cells. Physics in Medicine and Biology, 52: 4009-4025.

Burg, T.P., Godin, M., Knudsen, S.M., Shen, W., Carlson, G., Foster, J.S., Babcock, K., Manalis, S.R. 2007. Weighing of biomolecules, single cells and single nanoparticles in fluid. Nature, 446: 1066-1069.

Calleja, M., Tamayo, J., Nordstrom, M., Boisen, A. 2006. Low-noise polymeric nanomechanical biosensors. Applied Physics Letters, 88: 113901113903.

Carrascosa, L.G., Moreno, M., Alvarez, M., Lechuga, L.M. 2006. Nanomechanical biosensors: a new sensing tool. TrAC-Trends in Analytical Chemistry, 25: 196-206.

Cattani-Scholz, A., Pedone, D., Dubey, M., Neppl, S., Nickel, B., Feulner, P., Schwartz, J., Abstreiter, G., Tornow, M. 2008. Organophosphonate-based PNAfunctionalization of silicon nanowires for labelfree DNA detection. ACS Nano, 2: 1653-1660.

Chang, K.S., Chen, C.C., Sheu, J.T., Li, Y.K. 2009. Detection of an uncharged steroid with a silicon nanowire field-effect transistor. Sensors and Actuators B:Chemical, 138: 148-153.

Chen, C.P., Ganguly, A., Wang, C.H., Hsu, C.W., Chattopadhyay, S., Hsu, Y.K., Chang, Y.C., Chen, K.H., Chen, L.C. 2009. Label-free dual sensing of DNA molecules using GaN nanowires. Analytical Chemistry, 81: 36-42.

Cheng, M.M.C., Cuda, G., Bunimovich, Y.L., Gaspari, M., Heath, J.R., Hill, H.D., Mirkin, C.A., Nijdam, A.J., Terracciano, R., Thundat, T., Ferrari, M. 2006. Nanotechnologies for biomolecular detection and medical diagnostics. Current Opinion in Chemical Biology, 10: 11-19.

Choi, Y., Moody, I.S., Sims, P.C., Hunt, S.R., Corso, B.L., Perez, I., Weiss, G.A., Collins, P.G. 2012. Single-molecule lysozyme dynamics monitored by an electronic circuit. Science, 335: 319-324.

Claussen, J.C., Franklin, A.D., ul Haque, A., Porterfield, D.M., Fisher, T.S. 2009. Electrochemical biosensor of nanocube-augmented carbon nanotube networks. ACS Nano, 3: 37-44.

Collings, A.F., Caruso, F. 1997. Biosensors: recent advances. Reports on Progress in Physics, 60: 1397-1445.
Cui, Y., Wei, Q.Q., Park, H.K., Lieber, C.M. 2001. Nanowire nanosensors for highly sensitive and selective detection of biological and chemical species. Science, 293: 1289-1292.

Curreli, M., Zhang, R., Ishikawa, F.N., Chang, H.K., Cote, R.J., Zhou, C., Thompson, M.E. 2008. Realtime, label-free detection of biological entities using nanowire-based FETs. IEEE Transactions on Nanotechnology, 7: 651-667.

Dhayal, B., Henne, W.A., Doorneweerd, D.D., Reifenberger, R.G., Low, P.S. 2006. Detection of Bacillus subtilis spores using peptide-functionalized cantilever arrays. Journal of the American Chemical Society, 128: 3716-3721.

Erickson, D., Mandal, S., Yang, A.H.J., Cordovez, B. 2008. Nanobiosensors optofluidic, electrical and mechanical approaches to biomolecular detection at the nanoscale. Microfluidics and Nanofluidics, 4: 33-52.

Fang, Z.C., Kelley, S.O. 2009. Direct electrocatalytic mRNA detection using PNA-nanowire sensors. Analytical Chemistry, 81: 612-617.

Fritz, J., Baller, M.K., Lang, H.P., Rothuizen, H., Vettiger, P., Meyer, E., Guntherodt, H.J., Gerber, C., Gimzewski, J.K. 2000. Translating biomolecular recognition into nanomechanics. Science, 288: 316318.

Gao, C., Guo, Z., Liu, J.H., Huang, X.J. 2012. The new age of carbon nanotubes: An updated review of functionalized carbon nanotubes in electrochemical sensors. Nanoscale, 4: 1948-1963.

Gao, Z.Q., Agarwal, A., Trigg, A.D., Singh, N., Fang, C., Tung, C.H., Fan, Y., Buddharaju, K.D., Kong, J.M. 2007. Silicon nanowire arrays for label-free detection of DNA. Analytical Chemistry, 79: 32913297.

Gfeller, K.Y., Nugaeva, N., Hegner, M. 2005. Rapid biosensor for detection of antibiotic-selective growth of Escherichia coli. Applied and Environmental Microbiology, 71: 2626-2631.

Godin, M., Bryan, A.K., Burg, T.P., Babcock, K., Manalis, S.R. 2007. Measuring the mass, density, and size of particles and cells using a suspended microchannel resonator. Applied Physics Letters, 91: 123121-123123.

Grayson, A.C.R., Shawgo, R.S., Johnson, A.M., Flynn, N.T., Li, Y.W., Cima, M.J., Langer, R. 2004. A BioMEMS review: MEMS technology for physiologically integrated devices. Proceedings of the IEEE, 92: 6-21. 
Grieshaber, D., MacKenzie, R., Voros, J., Reimhult, E. 2008. Electrochemical biosensors-sensor principles and architectures. Sensors, 8: 1400-1458.

Grover, W.H., Bryan, A.K., Diez-Silva, M., Suresh, S., Higgins, J.M., Manalis, S.R. 2011. Measuring single-cell density. Proceedings of the National Academy of Sciences of the United States of America, 108: 10992-10996.

Gruhl, F.J., Rapp, B.E., Länge, K. 2013. Biosensors for diagnostic applications, Advances in Biochemical Engineering/Biotechnology, 133: 115-148.

Gruner, G. 2006. Carbon nanotube transistors for biosensing applications. Analytical and Bioanalytical Chemistry, 384: 322-335.

Gupta, A., Akin, D., Bashir, R. 2004. Detection of bacterial cells and antibodies using surface micromachined thin silicon cantilever resonators. Journal of Vacuum Science \& Technology B, 22: 2785-2791.

He, B., Morrow, T.J., Keating, C.D. 2008. Nanowire sensors for multiplexed detection of biomolecules. Current Opinion in Chemical Biology, 12: 522528.

Hyun, S.-J., Kim, H.-S., Kim, Y.-J., Jung, H.-I. 2006. Mechanical detection of liposomes using piezoresistive cantilever. Sensors and Actuators B: Chemical, 117: 415-419.

Icoz, K., Savran, C. 2010. Nanomechanical biosensing with immunomagnetic separation. Applied Physics Letters, 97: 123701-123703.

Icoz, K., Iverson, B.D., Savran, C. 2008. Noise analysis and sensitivity enhancement in immunomagnetic nanomechanical biosensors. Applied Physics Letters, 93: 103902-103904.

Ilic, B., Czaplewski, D., Craighead, H.G., Neuzil, P., Campagnolo, C., Batt, C. 2000. Mechanical resonant immunospecific biological detector. Applied Physics Letters, 77: 450-452.

Ishikawa, F.N., Chang, H.K., Curreli, M., Liao, H.I., Olson, C.A., Chen, P.C., Zhang, R., Roberts, R.W., Sun, R., Cote, R.J., Thompson, M.E., Zhou, C.W. 2009. Label-free, electrical detection of the SARS virus N-protein with nanowire biosensors utilizing antibody mimics as capture probes. ACS Nano, 3: 1219-1224.

Johnson, L., Gupta, A.T.K., Ghafoor, A., Akin, D., Bashir, R. 2006. Characterization of Vaccinia virus particles using microscale silicon cantilever resonators and atomic force microscopy. Sensors and Actuators B: Chemical, 115: 189-197.

Kerman, K. 2008. Nanomaterial-based electrochemical biosensors for medical applications. TrAC-Trends in Analytical Chemistry, 27: 585-592.

Kim, K.S., Lee, H.S., Yang, J.A., Jo, M.H., Hahn, S.K. 2009. The fabrication, characterization and application of aptamer-functionalized Si-nanowire FET biosensors. Nanotechnology, 20: 235501.

Kim, A., Ah, C.S., Yu, H.Y., Yang, J.H., Baek, I.B., Ahn, C.G., Park, C.W., Jun, M.S., Lee, S. 2007. Ultrasensitive, label-free, and real-time immunodetection using silicon field-effect transistors. Applied Physics Letters, 91: 103901-103903.

Kojima, A., Hyon, C.K., Kamimura, T., Maeda, M., Matsumoto, K. 2005. Protein sensor using carbon nanotube field effect transistor. Japanese Journal of Applied Physics, 44: 1596.

Lee, D., Chander, Y., Goyal, S.M., Cui, T. 2011. Carbon nanotube electric immunoassay for the detection of swine influenza virus H1N1. Biosensors \& Bioelectronics, 26: 3482-3487.

Lee, H.S., Kim, K.S., Kim, C.J., Hahn, S.K., Jo, M.H. 2009. Electrical detection of VEGFs for cancer diagnoses using anti-vascular endotherial growth factor aptamer-modified Si nanowire FETs. Biosensors \& Bioelectronics, 24: 1801-1805.

Li, C., Curreli, M., Lin, H., Lei, B., Ishikawa, F.N., Datar, R., Cote, R.J., Thompson, M.E., Zhou, C.W. 2005. Complementary detection of prostate-specific antigen using $\ln (2) \mathrm{O}(3)$ nanowires and carbon nanotubes. Journal of the American Chemical Society, 127: 12484-12485.

Li, J., Ng, H.T., Cassell, A., Fan, W., Chen, H., Ye, Q., Koehne, J., Han, J., Meyyappan, M. 2003. Carbon nanotube nanoelectrode array for ultrasensitive DNA detection. Nano Letters, 3: 597-602.

Lin, C H., Hung, C.H., Hsiao, C.Y., Lin, H.C., Ko, F.H., Yang, Y.S. 2009. Poly-silicon nanowire field-effect transistor for ultrasensitive and label-free detection of pathogenic avian influenza DNA. Biosensors \& Bioelectronics, 24: 3019-3024.

Lu, Y.S., Yang, M.H., Qu, F.L., Shen, G.L., Yu, R.Q. 2007. Amperometric biosensors based on platinum nanowires. Analytical Letters, 40: 875-886.

Maehashi, K., Katsura, T., Kerman, K., Takamura, Y., Matsumoto, K., Tamiya, E. 2007. Label-free protein biosensor based on aptamer-modified carbon nanotube field-effect transistors. Analytical Chemistry, 79: 782-787.

Manalis, S.R., Minne, S.C., Atalar, A., Quate, C.F. 1996. Interdigital cantilevers for atomic force microscopy. Applied Physics Letters, 69: 3944-3946. 
Martinez, J.A., Misra, N., Wang, Y.M., Stroeve, P., Grigoropoulos, C.P., Noy, A. 2009. Highly efficient biocompatible single silicon nanowire electrodes with functional biological pore channels. Nano Letters, 9: 1121-1126.

McKendry, R., Zhang, J.Y., Arntz, Y., Strunz, T., Hegner, M., Lang, H.P., Baller, M.K., Certa, U., Meyer, E., Guntherodt, H.J., Gerber, C. 2002. Multiple labelfree biodetection and quantitative DNA-binding assays on a nanomechanical cantilever array. Proceedings of the National Academy of Sciences of the United States of America, 99: 9783-9788.

Moore, D.F., Syms, R.R.A. 1999. Recent developments in micromachined silicon. Electronics \& Communication Engineering Journal, 11: 261-270.

Murphy, L. 2006. Biosensors and bioelectrochemistry. Current Opinion in Chemical Biology, 10: 177-184.

Musameh, M.M., Gao, Y., Hickey, M., Kyratzis, I.L. 2012. Application of carbon nanotubes in the extraction and electrochemical detection of organophosphate pesticides: A review. Analytical Letters, 45: 783-803.

Neuberger, T., Schopf, B., Hofmann, H., Hofmann, M., von Rechenberg, B. 2005. Superparamagnetic nanoparticles for biomedical applications. Possibilities and limitations of a new drug delivery system. Journal of Magnetism and Magnetic Materials, 293: 483-496.

Nicu, L., Leichle, T. 2008. Biosensors and tools for surface functionalization from the macro-to the nanoscale. The way forward. Journal of Applied Physics, 104: 101-111.

Oh, J., Chang, Y.W., Kim, H.J., Yoo, S., Kim, D.J., Im, S., Park, Y.J., Kim, D., Yoo, K.-H. 2010. Carbon nanotube-based dual-mode biosensor for electrical and surface plasmon resonance measurements. Nano Letters, 10: 2755-2760.

Onaran, A.G., Balantekin, M., Lee, W., Hughes, W.L., Buchine, B.A., Guldiken, R.O., Parlak, Z., Quate, C.F., Degertekin, F.L. 2006. A new atomic force microscope probe with force sensing integrated readout and active tip. Review of Scientific Instruments, 77: 023501-7.

Park, K., Millet, L.J., Kim, N., Li, H., Jin, X., Popescu, G., Aluru, N.R., Hsia, K.J., Bashir, R. 2010. Measurement of adherent cell mass and growth. Proceedings of the National Academy of Sciences of the United States of America, 107: 20691-20696.

Patolsky, F., Timko, B.P., Yu, G.H., Fang, Y., Greytak, A.B., Zheng, G.F., Lieber, C.M. 2006. Detection, stimulation, and inhibition of neuronal signals with high-density nanowire transistor arrays. Science, 313: 1100-1104.

Patolsky, F., Zheng, G., Lieber, C.M. 2006. Nanowire sensors for medicine and the life sciences. Nanomedicine, 1: 51-65.

Patolsky, F., Zheng, G.F., Hayden, O., Lakadamyali, M., Zhuang, X.W., Lieber, C.M. 2004. Electrical detection of single viruses. Proceedings of the National Academy of Sciences of the United States of America, 101: 14017-14022.

Pei, J.H., Tian, F., Thundat, T. 2004. Glucose biosensor based on the microcantilever. Analytical Chemistry, 76: 292-297.

Pui, T.S., Agarwal, A., Ye, F., Balasubramanian, N., Chen, P. 2009. CMOS-compatible nanowire sensor arrays for detection of cellular bioelectricity. Small, 5: 208-212.

Raiteri, R., Grattarola, M., Butt, H.J., Skladal, P. 2001. Micromechanical cantilever-based biosensors. Sensors and Actuators B: Chemical, 79: 115-126.

Savran, C.A., Knudsen, S.M., Ellington, A.D., Manalis, S.R. 2004. Micromechanical detection of proteins using aptamer-based receptor molecules. Analytical Chemistry, 76: 3194-3198.

Sharf, T., Kevek, J.W., DeBorde, T., Wardini, J.L., Minot, E.D. 2012. Origins of charge noise in carbon nanotube field-effect transistor biosensors. Nano Letters, 12: 6380-6384.

Shoorideh, K., Chi On, C. 2012. Optimization of the sensitivity of FET-based biosensors via biasing and surface charge engineering. IEEE Transactions on Electron Devices, 59: 3104-3110.

Sone, H., Ikeuchi, A., Izumi, T., Okano, H., Hosaka, S. 2006. Femtogram mass biosensor using self-sensing cantilever for allergy check. Japanese Journal of Applied Physics, 45: 2301-2304.

Sorgenfrei, S., Chiu, C.-y., Gonzalez, R.L., Yu, Y.-J., Kim, P., Nuckolls, C., Shepard, K.L. 2011. Labelfree single-molecule detection of DNA-hybridization kinetics with a carbon nanotube field-effect transistor. Nature Nanotechnology, 6: 126-132.

Staples, M., Daniel, K., Cima, M.J., Langer, R. 2006. Application of micro- and nanoelectromechanical devices to drug delivery. Pharmaceutical Research, 23: 847-863.

Stern, E., Vacic, A., Reed, M.A. 2008. Semiconducting nanowire field-effect transistor biomolecular sensors. IEEE Transactions on Electron Devices, 55: 3119-3130. 
Stern, E., Klemic, J.F., Routenberg, D.A., Wyrembak, P.N., Turner-Evans, D.B., Hamilton, A.D., LaVan, D.A., Fahmy, T.M., Reed, M.A. 2007. Label-free immunodetection with CMOS-compatible semiconducting nanowires. Nature, 445: 519-522.

Stoney, G.G. 1909. The tension of metallic films deposited by electrolysis. Proceedings of the Royal Society of London Series A, Mathematical, Physical Engineering Sciences, 82: 172-175.

Storri, S., Santoni, T., Minunni, M., Mascini, M. 1998. Surface modifications for the development of piezoimmunosensors. Biosensors \& Bioelectronics, 13: 347-357.

Strehlitz, B., Nikolaus, N., Stoltenburg, R. 2008. Protein detection with aptamer biosensors. Sensors, 8: 4296-4307.

Sulchek, T., Hsieh, R., Minne, S.C., Quate, C.F., Manalis, S.R. 2001. Interdigital cantilever as a biological sensor. In: Proceedings of the $1^{\text {st }}$ IEEE Conference on Nanotechnology, pp. 562-566.

Tang, T., Liu, X.L., Li, C., Lei, B., Zhang, D.H., Rouhanizadeh, M., Hsiai, T., Zhou, C.W. 2005. Complementary response of $\operatorname{In}_{2} \mathrm{O}_{3}$ nanowires and carbon nanotubes to low- density lipoprotein chemical gating. Applied Physics Letters, 86: 103903.

Tolani, S.B., Craig, M., DeLong, R.K., Ghosh, K., Wanekaya, A.K. 2009. Towards biosensors based on conducting polymer nanowires. Analytical and Bioanalytical Chemistry, 393: 1225-1231.

Wanekaya, A.K., Chen, W., Myung, N.V., Mulchandani, A. 2006. Nanowire-based electrochemical biosensors. Electroanalysis, 18: 533-550.

Wang, J. 2006. Electrochemical biosensors. Towards point-of-care cancer diagnostics. Biosensors \& Bioelectronics, 21: 1887-1892.

Wang, R.H., Ruan, C.M., Kanayeva, D., Lassiter, K., Li, Y.B. 2008. $\mathrm{TiO}_{2}$ nanowire bundle microelectrode based impedance immunosensor for rapid and sensitive detection of Listeria monocytogenes. Nano Letters, 8: 2625-2631.

Wang, X., Ozkan, C.S. 2008. Multisegment nanowire sensors for the detection of DNA molecules. Nano Letters, 8: 398-404.

Weizmann, Y., Elnathan, R., Lioubashevski, O., Willner, I. 2005. Magnetomechanical detection of the specific activities of endonucleases by cantilevers. Nano Letters, 5: 741-744.

Weizmann, Y., Patolsky, F., Lioubashevski, O., Willner, I. 2004. Magneto-mechanical detection of nucleic acids and telomerase activity in cancer cells. Journal of the American Chemical Society, 126: 1073-1080.

Wu, G.H., Ji, H.F., Hansen, K., Thundat, T., Datar, R., Cote, R., Hagan, M.F., Chakraborty, A.K., Majumdar, A. 2001. Origin of nanomechanical cantilever motion generated from biomolecular interactions. Proceedings of the National Academy of Sciences of the United States of America, 98: 1560-1564.

Yaralioglu, G.G., Atalar, A., Manalis, S.R., Quate, C.F. 1998. Analysis and design of an interdigital cantilever as a displacement sensor. Journal of Applied Physics, 83: 7405-7415.

Yeom, S.-H., Kang, B.-H., Kim, K.-J., Kang, S.-W. 2011. Nanostructures in biosensor, a review. Frontiers in Bioscience, 16: 997-1023.

Zhang, F.Y., Ulrich, B., Reddy, R.K., Venkatraman, V.L., Prasad, S., Vu, T.Q., Hsu, S.T. 2008. Fabrication of submicron $\mathrm{IrO}_{2}$ nanowire array biosensor platform by conventional complementary metaloxide-semiconductor process. Japanese Journal of Applied Physics, 47: 1147-1151.

Zhang, G.-J., Zhang, L., Huang, M.J., Luo, Z.H.H., Tay, G.K.I., Lim, E.-J.A., Kang, T.G., Chen, Y. 2010. Silicon nanowire biosensor for highly sensitive and rapid detection of Dengue virus. Sensors and Actuators B: Chemical, 146: 138-144.

Zhang, G.J., Chua, J.H., Chee, R.E., Agarwal, A., Wong, S.M. 2009. Label-free direct detection of MiRNAs with silicon nanowire biosensors. Biosensors \& Bioelectronics, 24: 2504-2508.

Zhang, J., Lang, H.P., Huber, F., Bietsch, A., Grange, W., Certa, U., McKendry, R., Guntgerodt, H.J., Hegner, M., Gerber, C. 2006. Rapid and label-free nanomechanical detection of biomarker transcripts in human RNA. Nature Nanotechnology, 1: 214-220.

Zhang, L.J., Peng, H., Kilmartin, P.A., Soeller, C., Travas-Sejdic, J. 2007. Polymeric acid doped polyaniline nanotubes for oligonucleotide sensors. Electroanalysis, 19: 870-875.

Zhang, X.Y., Li, D., Bourgeois, L., Wang, H.T., Webley, P.A. 2009. Direct electrodeposition of porous gold nanowire arrays for biosensing applications. Chemphyschem, 10: 436-441.

Zheng, G.F., Patolsky, F., Cui, Y., Wang, W.U., Lieber, C.M. 2005. Multiplexed electrical detection of cancer markers with nanowire sensor arrays. Nature Biotechnology, 23: 1294-1301.

Ziegler, C. 2004. Cantilever-based biosensors. Analytical and Bioanalytical Chemistry, 379: 946-959. 